\title{
Discrete-time nonlinear sliding mode controller
}

\author{
Nikhil Kumar Yadav $^{1}$, R.K. Singh ${ }^{2}$ \\ ${ }^{1 *}$ Department of Electrical Engineering, Motilal Nehru National Institute of Technology Allahabad(UP), INDIA \\ ${ }^{2}$ Department of Electrical Engineering, Motilal Nehru National Institute of Technology Allahabad(UP), INDIA \\ ${ }^{1 *}$ Corresponding Author: e-mail: nyadav24@gmail.com ,Mob-09818486179
}

\begin{abstract}
The objective of this paper is to design nonlinear sliding surface with investigation of the state delays. A particular linear transformation is defined to transform the time-delay and stability is proven for nonlinear sliding surface, which leads to show the asymptotic stability of the system.
\end{abstract}

Keywords: Discrete-time delay system, Sliding mode control, nonlinear sliding surface, optimal control.

\section{Introduction}

The time delay is a quite common in all types of engineering systems such as chemical process control, delay in the actuator operation of mechanical systems and economic systems etc. Compared to the systems without delay, the presence of the delay makes the system more difficult to achieve its satisfactory performance of the systems. In recent years there is a great interest of researchers for processes having the state delays.

The concept of the sliding mode control in recent years has drawn the attention of researchers (Bartoszewicz, 1998; Utkin, 1977; Gao. 1977; Lee et al., 2001). Sliding mode control is a methodology in which an appropriate input is provided so that the system states are confined to the desired sub-manifold of the state space. Furuta (1990) proposed discrete time sliding mode control laws where the control input includes state feedback terms in three different feedback coefficients. The discrete time sliding mode control has been mainly applied to stabilization problems. However in stabilization problems only bounded stability is guaranteed if the uncertainties in the system do not vanish at the equilibrium points. Won and Hedrick (1995) deal with the mismatch uncertainties for discrete adaptive sliding mode control for the nonlinear system for the output to track a sufficiently smooth desired trajectory. In Bandhyopadhyay and Deepak (2009), nonlinear sliding surface is created for varying the damping factor for improving the performance of the multi-input and multi-output linear systems with matched conditions. Some of the concepts and theoretical advances of continuous time sliding mode control are covered in literature (Utkin, 1976; Hung et al., 1993; El.Ghezawi et al., 1983; Young et al., 1999; Edward and Spurgeon, 1998) and the references therein. Due to flexibility of implementation, large classes of continuous systems are controlled by digital signal processors and microprocessors. To analyze the sampling time effect, discrete sliding mode control is well studied in the literature (Gao et al., 1995a,b; Spurgeon, 1992; Golo and Milosavljevic, 2000; Hu et al., 2009; Furukawa and Shimemura, 1983; Janardhanan and Bandhyopadhyay, 2006; Fiagbedzi and Pearson, 1986; Jafarov, 2000; Sarpturk et al., 1987). This paper has been organized as follows: Section II briefly introduces state about the description of the discrete -time plant with state delay and its transformation, section III illustrates proposed design of nonlinear sliding surface, section IV illustrates result analysis and section V describes the conclusion.

\section{Problem Statement:}

Consider the following discrete time-delay systems:

$$
\begin{aligned}
& x(k+1)=\bar{A} x(k)+\bar{A}_{d} x(k-d)+\bar{B} u(k) \\
& y(k)=\bar{C} x(k)
\end{aligned}
$$


Where $x(k) \in R^{n}$ is the state vector, $u(k) \in R^{m}$ is the control input, $y(k) \in R^{p}$ is the output respectively. The term $\bar{A}, \bar{A}_{d}, \bar{B}$ and $\bar{C}$ are some constant matrices of appropriate dimensions and parameter $d>0$ is a constant.

We assume the following assumptions.

Assumption I: the pair $(\bar{A}, \bar{B})$ is controllable, the pair $(\bar{A}, \bar{C})$ is observable, matrices $\bar{B}$ and $\bar{C}$ have full rank.

Assumption II: $\mathrm{p}>\mathrm{m}$ and $\operatorname{rank}(-\bar{C} \bar{B})=\mathrm{m}$.

For analyzing the system (1), we will transform it in to its regular form. Let suppose $B=\left[\begin{array}{ll}B_{1} & B_{2}\end{array}\right]^{T}$

And satisfies the det $\left(B_{2}\right) \neq 0$, there exists a linear non-singular transformation

$$
z=T X=\left[\begin{array}{cc}
I_{n-m} & -B_{1} B_{2}^{-1} \\
0 & B_{2}^{-1}
\end{array}\right] X
$$

It transforms the system dynamics (1) to its regular form

$$
\begin{aligned}
& z(k+1)=A z(k)+A_{d} z(k-d)+B u(k) \\
& y(k)=C z(k)
\end{aligned}
$$

Where

$$
z(k)=\left[\begin{array}{l}
z_{1}(k) \\
z_{2}(k)
\end{array}\right]
$$

The transform of the system can be shown with out loss of generality.

$$
\begin{aligned}
& z_{1}(k+1)=A_{11} z_{1}(k)+A_{12} z_{2}(k)+A_{d 11} z_{1}(k-d)+A_{d 12} z_{2}(k-d) \\
& z_{2}(k+1)=A_{11} z_{1}(k)+A_{22} z_{2}(k)+A_{d 21} z_{1}(k-d)+A_{d 22} z_{2}(k-d)+B_{2} u(k) \\
& y(k)=C z(k)
\end{aligned}
$$

Where $z_{1}(k) \in R^{(n-m)}, \quad z_{2}(k) \in R^{m}$ are the state vectors and the matrices $\mathrm{A}, \mathrm{A}_{\mathrm{d}}, \mathrm{B}_{2}$ are the constant , with their appropriate dimensions. The following matrix can be written as follows after transformation. Regular form of the equation can be written as follows

$$
\begin{aligned}
& A=T \bar{A} T^{-1}=\left[\begin{array}{ll}
A_{11} & A_{12} \\
A_{21} & A_{22}
\end{array}\right] \\
& A_{\text {reg }}=\left[\begin{array}{ll}
A_{11} & A_{12} \\
A_{21} & A_{22}
\end{array}\right]
\end{aligned}
$$

The regular form of the system's state delay is as follows

$$
\begin{gathered}
A_{d}=T \bar{A}_{d} T^{-1}=\left[\begin{array}{ll}
A_{d 11} & A_{d 12} \\
A_{d 21} & A_{d 22}
\end{array}\right] \\
A_{d r e g}=\left[\begin{array}{ll}
A_{d 11} & A_{d 12} \\
A_{d 21} & A_{d 22}
\end{array}\right] \\
B=T \quad \bar{B}\left[\begin{array}{c}
0 \\
I_{m}
\end{array}\right] \\
C=\bar{C} T^{-1}
\end{gathered}
$$

$Z_{1} \in \mathfrak{R}^{n-m}$ and $Z_{2} \in \mathfrak{R}^{m}$ are the state vectors. $A_{11}, A_{12}, A_{21}, A_{22}$ are known constant matrices with appropriate dimensions. $I_{m}$ is $m \times m$ identity matrix.

2.1 Calculating the optimal gain matrix : From the discrete-time delay system, the gain matrix can be calculated as follows:

To Minimized the cost function

$$
\begin{aligned}
& x(k+1)=\bar{A} x(k)+\overline{A_{d}} x(k-d)+\bar{B} u(k) \\
& y(k)=\bar{C} x(k)
\end{aligned}
$$

$$
J=\frac{1}{2} \sum_{0}^{\infty}\left(x^{T}(k) Q x(k)+u^{T}(k) R u(k)\right)
$$


To find out the gain with the help of LQR method and the resulting optimal gain matrix

$\mathrm{R}=1$,

$\mathrm{Q}=\operatorname{diag}(\mathrm{Q} 1, \mathrm{Q} 2, \mathrm{Q})$

$\mathrm{G}=\operatorname{lqr}(\bar{A}, \bar{B}, \mathrm{Q}, \mathrm{R})$

\section{Designing of Nonlinear Sliding Surface}

In this section the designing of the nonlinear sliding surfaces for the general MIMO systems. Without loss of generality, the system described by (1) can be transformed in to regular form as follows

$$
\begin{aligned}
& z_{1}(k+1)=A_{11} z_{1}(k)+A_{12} z_{2}(k)+A_{d 11} z_{1}(k-d)+A_{d 12} z_{2}(k-d) \\
& z_{2}(k+1)=A_{11} z_{1}(k)+A_{22} z_{2}(k)+A_{d 21} z_{1}(k-d)+A_{d 22} z_{2}(k-d)+B_{2} u(k) \\
& y(k)=C z(k) \\
& z=T x=\left[\begin{array}{c}
z_{1}(k) \\
z_{2}(k)
\end{array}\right]
\end{aligned}
$$

The sliding surface for the system in regular form is proposed as follows

$$
S(k)=C^{T} z(k)=0
$$

Without loss of generality of the system, the sliding mode control is as follows

$$
S(k)=C^{T} z(k)=\left[\begin{array}{ll}
C_{1} & I_{m}
\end{array}\right]\left[\begin{array}{l}
z_{1}(k) \\
z_{2}(k)
\end{array}\right]
$$

Where $C^{T} \in \mathfrak{R}^{m \times n}, C_{1} \in \mathfrak{R}^{m \times(n-m)}, I_{m} \in \mathfrak{R}^{m \times m}$ dimension. For sliding motion the sliding condition is as follows

Then

$$
S(k)=C^{T} z(k)=0
$$

$$
\begin{gathered}
S(k)=C^{T} z(k)=0 \\
S(k)=\left[\begin{array}{ll}
C_{1} & I_{m}
\end{array}\right]\left[\begin{array}{l}
z_{1}(k) \\
z_{2}(k)
\end{array}\right]=0 \\
S(k)=C_{1} z_{1}(k)+z_{2}(k)=0 \\
z_{2}(k)=-C_{1} z_{1}(k) \\
S(k)=\left[\begin{array}{lll}
\left.G-\psi(y(k))\left\{A_{12}^{T} P\left(A_{11}-A_{12} G\right)\right)\right\} & I_{m}
\end{array}\right]\left[\begin{array}{c}
z_{1}(k) \\
z_{2}(k)
\end{array}\right] \\
C_{1}=\left[\begin{array}{lll}
G & -\psi(y(k))\left\{A_{12}^{T} P\left(A_{11}-A_{12} G\right)\right\}
\end{array}\right] \\
z_{1}(k+1)=A_{11 \text { eq }} z_{1}(k)+A_{12} \psi(y(k)) A_{12}^{T} P A_{11 \text { eq }} z_{1}(k)
\end{gathered}
$$

Seeing $z_{2}(k)$ as dummy input of the subsystem (9). Substituting (12) into subsystem (9) yields

$$
\begin{aligned}
& z_{1}(k+1)=A_{11} z_{1}(k)-A_{12} C_{1} z_{1}(k)+A_{d 11} z_{1}(k-d)-A_{d 12} C_{1} z_{2}(k-d) \\
& z_{1}(k+1)=\left(A_{11}-A_{12} C_{1}\right) z_{1}(k)+\left(A_{d 11}-A_{d 12} C_{1}\right) z_{1}(k-d)
\end{aligned}
$$

The nonlinear sliding surface can be designed by incorporating the nonlinearity $\psi(y(k))$ in the system dynamics for proven for its stability. If the nonlinear surface is stable then the all the states trajectory will be on the stable surface for future all the time. The condition for the stability of the nonlinear surface as follows

$$
2 \psi(y(k))+\psi(y(k)) A_{12}^{T} P A_{12} \psi(y(k)) \leq 0
$$

3.1 Proof: The proof for the nonlinear sliding surface as stability follows.

Consider Lyapunov functional candidate for proving the nonlinear sliding surface as stable

$$
\begin{gathered}
V_{1}(k)=z_{1}^{T}(k) P z_{1}(k) \\
\Delta V(k)=V(k+1)-V(k) \\
\Delta V_{1}(k)=z_{1}^{T}(k+1) P z_{1}(k+1)-z_{1}^{T}(k) P z_{1}(k)
\end{gathered}
$$




$$
\begin{aligned}
z_{1}(k+1) & =A_{11} z_{1}(k)+A_{12}\left(-C_{1} z_{1}(k)\right) \\
z_{1}(k+1) & =\left(A_{11}-A_{12} C_{1}\right) z_{1}(k) \\
& =A_{11}-A_{12}\left(G-\psi(y(k)) A_{12}^{T} P\left(A_{11}-A_{12} G\right)\right) z_{1}(k) \\
& =A_{11 e q} z_{1}(k)+A_{12} \psi(y(k)) A_{12}^{T} P A_{11 e q} z_{1}(k)
\end{aligned}
$$

Where $A_{11 e q}=\left(A_{11}-A_{12} G\right)$ and transform state dynamics becomes as follows

$$
\begin{aligned}
& z_{1}(k+1)=A_{11 e q} z_{1}(k)+A_{12} \psi(y(k)) A_{12}^{T} P A_{11 e q} z_{1}(k) \\
& S(k)=\left[\begin{array}{ll}
\left.G-\psi(y(k))\left\{A_{12}^{T} P\left(A_{11}-A_{12} G\right)\right)\right\} & I_{m}
\end{array}\right] \times\left[\begin{array}{c}
z_{1}(k) \\
z_{2}(k)
\end{array}\right] \\
& C_{1}=\left[G-\psi(y(k))\left\{A_{12}^{T} P\left(A_{11}-A_{12} G\right)\right\}\right] \\
& z_{1}(k+1)=A_{11 e q} z_{1}(k)+A_{12} \psi(y(k)) A_{12}^{T} P A_{11 e q} z_{1}(k)
\end{aligned}
$$

For stability ( $A_{11}-A_{12} G$ ) must have all the eigenvalues inside the unit circle. Now, we have to prove that the nonlinear sliding surface is stable by the Lyapunov method for the present state.

3.2 Proof : Consider the Lyapunov functional candidate as follows for proving asymptotic stability of the transform system

$$
\begin{gathered}
V_{1}(k)=z_{1}^{T}(k) P z_{1}(k) \\
V_{1}(k+1)=z_{1}^{T}(k+1) P z_{1}(k+1) \\
\Delta V(k)=z_{1}^{T}(k+1) P z_{1}(k+1)-z_{1}^{T}(k) P z_{1}(k) \\
\Delta V(k)=\left\{A_{11 e q} z_{1}(k)+A_{12} \psi(y(k)) A_{12}^{T} P A_{11 e q} z_{1}(k)\right\}^{T} P *\left\{A_{11 e q} z_{1}(k)+A_{12} \psi(y(k)) A_{12}^{T} P A_{11 e q} z_{1}(k)\right\} \\
-z_{1}^{T}(k) P z_{1}(k) \quad z_{1}^{T}(k) A_{11 e q}^{T} P A_{12} \psi(y(k)) A_{12}^{T} P A_{11 e q} z_{1}(k)+z_{1}^{T}(k) A_{11 e q}^{T} z_{1}(k)-z_{1}^{T}(k) P z_{1}(k) \\
+z_{1}^{T}(k) A_{11 e q}^{T} P A_{12} \psi(y(k)) A_{12}^{T} P A_{11 e q} z_{1}(k)+e_{1}^{T}(k) A_{11 e q}^{T} P A_{12} A_{12}^{T} \Psi(y(k)) \\
* A_{12}^{T} P A_{11 e q} e_{1}(k) \\
=-z_{1}^{T} H z_{1}(k)+z_{1}^{T}(k) A_{11 e q}^{T} P A_{12}\left\{2 \psi(y(k))+\psi(y(k)) A_{12}^{T} P A_{12} \psi(y(k))\right\} \\
* A_{12}^{T} P A_{11 e q} z_{1}(k)
\end{gathered}
$$

Where $\mathrm{H}$ is some small positive definite matrix

Let $z_{1}^{T}(k) A_{11 e q}^{T} P A_{12}=N^{T}$

$$
\Delta V_{1}(k)=N^{T}\left\{2 \psi(y(k))+\psi(y(k+)) A_{12}^{T} P A_{12} \psi(y(k+))\right\} N-z_{1}^{T}(k) H z_{1}(k)
$$

From (14)

$$
\begin{gathered}
2 \psi(y(k))+\psi(y(k)) A_{12}^{T} P A_{12} \psi(y(k)) \leq 0 \\
\Delta V(k)=-z_{1}^{T}(k) H z_{1}(k)
\end{gathered}
$$

Here it is proven that the nonlinear sliding surface is asymptotically stable as the $\Delta V(k)$ is negative definite, therefore all the states will be tracking on stable nonlinear sliding surface for all the future time.

3.3 Lemma 1 [22] Given constant matrices D, E and a symmetric constant matrix Y of appropriate dimensions, the following inequality holds:

$$
Y+D E F+E^{T} F^{T} D^{T}<0
$$

Where F satisfies $F^{T} F \leq N$, if and only if for some $\zeta>0$

$$
Y+\left[\begin{array}{ll}
\zeta^{-1} E^{T} & \zeta D
\end{array}\right]\left[\begin{array}{cc}
N & 0 \\
0 & I
\end{array}\right]\left[\begin{array}{l}
\zeta^{-1} E \\
\zeta D^{T}
\end{array}\right]<0 \quad \text { holds. }
$$

The main result of the asymptotic stability of the reduced order with time delay is summarized as per following theorem.

3.4 Theorem 1: If there exists a symmetric and positive definite matrix $\mathrm{P}$, some matrix $\mathrm{M}$ and some scalar $\zeta$ such that reduced order discrete-time system is asymptotically stable via sliding mode surface (13): 


$$
\left[\begin{array}{cccc}
-H & * & * & * \\
A_{11} H+A_{12} M & -H & * & * \\
E_{1} H+E_{2} M & 0 & -\zeta I & * \\
0 & \zeta D^{T} & 0 & -\zeta I
\end{array}\right]
$$

Where $-H=P^{-1}, M=C_{1} P^{-1}$ and $*$ denotes the transposed elements in the symmetric positions.

3.5 Proof: Consider the Lyapunov function candidate for delay system

$$
V(k)=z_{1}^{T}(k) P z_{1}(k)+\sum_{\alpha=k-d}^{k-1} z_{1}^{T}(\alpha) Q z_{1}(\alpha)
$$

Where P and Q are positive definite symmetrical matrices. The difference of $\mathrm{V}(\mathrm{k})$ is

$$
\begin{aligned}
& \Delta V(k)=V(k+1)-V(k) \\
& =z_{1}^{T}(k+1) P z_{1}(k+1)+\sum_{\alpha=k+1-d}^{k} z_{1}^{T}(\alpha) Q z_{1}(\alpha) \\
& -z_{1}^{T}(k) P z_{1}(k)-\sum_{\alpha=k-d}^{k-1} z_{1}^{T}(\alpha) Q z_{1}(\alpha) \\
& \Delta V(k)=\left[A_{1}^{T} z(k)+A_{d 1}^{T} z(k-d)\right]^{T} \times P\left[A_{1}^{T} z(k)+A_{d 1}^{T} z(k-d)\right] \\
& +z^{T}(k) Q z(k)-z^{T}(k-d) Q z(k-d) \\
& =\Psi^{T}(k)\left[\begin{array}{ll}
A_{1}^{T} & A_{d 1}^{T}
\end{array}\right]^{T} P\left[\begin{array}{ll}
A_{1}^{T} & A_{d 1}^{T}
\end{array}\right] \Psi(k)-\Psi^{T}(k)\left[\begin{array}{cc}
P-Q & 0 \\
0 & Q
\end{array}\right] \Psi(k) \\
& \Psi(k)=\left[\begin{array}{ll}
z_{1}^{T}(k) & z_{1}^{T}(k-d)
\end{array}\right]^{T} \\
& \Psi_{1}^{T}(k)\left(\left[\begin{array}{ll}
A_{1} & A_{d 1}
\end{array}\right]^{T} P\left[\begin{array}{ll}
A_{1} & A_{d 1}
\end{array}\right]+K\right) \Psi_{1}(k)
\end{aligned}
$$

Where

Where

$$
\begin{gathered}
\Psi(k)=\left[\begin{array}{c}
z_{1}(k) \\
z_{1}(k-d)
\end{array}\right] \\
K=\left[\begin{array}{cc}
Q-P & 0 \\
0 & -Q
\end{array}\right] \\
A_{1}=A_{11}-A_{12} G \\
A_{d 1}=A_{d 11}-A_{d 12} G
\end{gathered}
$$

Therefore sliding mode dynamics (13) is asymptotically stable, if the following inequality holds

$$
\left(\left[\begin{array}{ll}
A_{1} & A_{d 1}
\end{array}\right]^{T} P\left[\begin{array}{ll}
A_{1} & A_{d 1}
\end{array}\right]+K\right)<0
$$

Apply Schur complements in (14) is equal to

$$
\left[\begin{array}{ccc}
Q-P & * & * \\
0 & -Q & * \\
A_{1} & A_{d 1} & -P^{-1}
\end{array}\right]<0 \quad \text { condition hold. }
$$

*Denotes the transposed elements in the symmetric position. It will be negative definite and the change in Lyapunov function will be negative definite i.e.

$$
\Delta V(k)<0, \quad z_{1}(k) \neq 0, \quad k=1,2, \ldots .
$$

Now the system will be asymptotically stable.

\section{Designing of Control Law}

The designing of the control law is based upon the stable nonlinear sliding mode control which already proven for its stability.

$$
\begin{aligned}
& S(k)=C^{T} z(k) \\
& S(k+1)=C^{T} z(k+1)
\end{aligned}
$$


Sliding mode condition

$$
\begin{gathered}
S(k+1)=S(k)=0 \\
z(k+1)=A z(k)+A_{d}(k-d)+B u(k) \\
C^{T} z(k+1)=C^{T}\left[A z(k)+A_{d}(k-d)+B u(k)\right]=0 \\
=C^{T} A z(k)+C^{T} A_{d}(k-d)+C^{T} B u(k) \\
C^{T} A z(k)+C^{T} A_{d}(k-d)+C^{T} B u(k)=0 \\
u(k)=\left(C^{T} B\right)^{-1}\left[C^{T} A z(k)+C^{T} A_{d}(k-d)\right]
\end{gathered}
$$

At the sampling instant of discrete-time system, the state trajectory will remain on the nonlinear sliding surface and system will be stable for ever in all the future time. It is proven that control law (21) drives the system trajectory on to the nonlinear sliding surface and maintains the trajectory on the sliding surface during subsequent time.

\section{Conclusion}

In this paper, nonlinear sliding mode controller has been designed for the time delay in the discrete-time system. The nonlinear systems are highly unstable and the proposed algorithm of nonlinear sliding mode controller will have more effectiveness for solving the nonlinear systems.

\section{References}

Bandhyopadhyay B., Deepak F., 2009. High performance tracking controller for discrete plant using nonlinear sliding surface, IEEE Transactions on Industrial Electronics, Vol.56, No.9.

Bartoszewicz. A,, 1998. Discrete sliding mode control strategies, IEEE Transactions Industrial Electronics, Vol. 45, pp. 633-637.

El.Ghezawi O.M.E., A.S.I.Zinober and S.A.Billings, 1983. Analysis and design of variable structure systems using a geometric, Int. J. Control, Vol.38, No.3, pp. 657-671, Sep,

Edward C. and Spurgeon S.K. 1998. Sliding Mode Control, Theory and Application London, U.K.: Taylor \& Francis.

Fiagbedzi Y. A. and A. E. Pearson, 1986. Feedback stabilization of linear autonomous time lag systems, IEEE Transactions on Automatic Control, Vol. 31, pp. 847-855.

Furuta K., 1990. Sliding mode control of a discrete systems, Systems and Control Letters, Vol. 14, No. 2, pp. 145-152.

Furukawa Y. and E. Shimemura, 1983. Predictive control for systems with time-delay, International Journal of Control, Vol. 37, pp. 399-412.

Gao W., Y. Wang and A. Homaifa, 1995a. Discrete-time variable structure control systems, IEEE Trans. Ind. Electronics, Vol. 42, No.2, pp.117-122, Apr,.

Golo G. and C. Milosavljevic, 2000. Robust discrete-time chattering free sliding mode control, Syst. Control Lett., Vol. 41, No. 1, pp. 19-28, Sep.

Gao W., Wang Y., Homaifa A., 1995b. Discrete-time variable structure control systems, IEEE Transaction of Industrial Electronics, Vol. 42, No. 2, pp. 117-122.

Gao W.. 1998. The theory and design of variable structure control’ Beijing science publishing company (in Chinese)

Janardhanan S. and B. Bandhyopadhyay, 2006. Output feedback discrete-time sliding mode control for time delay systems, IEE Proc. Control Theory Application, Vol. 153, No. 4, July.

Hu Q., Du C., Xie L., Wang Y, 2009. Discrete-time sliding mode control with time-varying surface for hard disk drives, IEEE Trans. Control System. Technology, Vol. 17, No. 1, pp. 175-183.

Hung J.Y., W. Gao and J.C.Hung, 1993. Variable structure control: A survey, IEEE Transactions Industrial Electronics, Vol.40, No.1, pp.2-22, Feb.

Jafarov E. M., 2000. Designing of sliding mode control for multi-inputs systems with multiple states delays”, Proc. of the America Control Conference, Vol.2, pp.1139-1143.

Lee H.J., Jin Bae Park and Guanrong Chen, 2001. Robust fuzzy control of nonlinear systems with parametric uncertainties, IEEE

Trans. Fuzzy system, Apr, pp. 369-379.

Sarpturk. S.Z., Istefanopulos. Y., and Kaynak..O.,1987. On the stability of discrete-time sliding mode systems, IEEE Transaction Automatic Control, AC-32, pp.930-932.

Spurgeon S.K., 1992. Hyper-plane design technology for discrete time variable structure control systems ,” Int. J. Cont., Vol. 55, No.2, pp. 445-456, Feb,

Utkin V.I., 1977. Variable structure control systems with sliding mode. IEEE Trans. On Automat. Control, Vol.22, pp. $212-222$.

Utkin V. I., 1976. Variable structure systems with sliding modes, IEEE transactions Automatic Control, Vol. AC-22, No.2 , pp. 212-221, Apr. 
Won M., Hedrick J.K., 1995. Discrete time adaptive sliding control of nonlinear systems without matching condition, American Control Conference Sealtle, Washington, pp. 1309-1313.

Young K.D., V.I. Utkin and U.Ozgunar, 1999. A control engineer's guide to sliding mode control, IEEE Trans. Control Syst. Technology, Vol. 7, No.3, pp. 328-342, May.

\section{Biographical notes}

Nikhil Kumar Yadav (S’09) was born in Etawah, Uttar Pradesh, India, on April 02, 1969. He received the AMIE degree in (Electronics and Telecommunication Engineering) from Institute of Engineers (India), Calcutta, India in 1992, MBA (Marketing \& Personnel) from University of Pune India 1997 \& 1999 respectively, M. Tech degree in electrical engineering with specialization in control and instrumentation from Motilal Nehru National Institute of Technology (MNNIT), Allahabad (Uttar Pradesh) India, in 2005. He is currently working towards the Ph.D. degree, M. N. National Institute of Technology, Allahabad (UP), India. He is also an Assistant Professor with Electrical and Electronics Engineering Department, Lingaya's University, Faridabad, (Haryana), India. His current research interests are in areas of variable structure control, nonlinear system dynamics and control, and robust control techniques.

Dr. R. K Singh, is a Professor in the Department of Electrical Engineering, Motilal Nehru National Institute of Technology, Allahabad (MNNIT) , India. He is having more than 20 years of teaching and research experience. His area of interest is dc-dc converters, electrical vehicle and distributed generation application. He is reviewer of national and international journal/conferences and working on research projects of government of India. Presently he is chairing IEEE local centre at MN National Institute of Technology, Allahabad. He is also a student's counselor of IET local chapter of M. N. National Institute of Technology Allahabad (UP) India. . He is a Fellow of IE (India) and member of IEEE.

Received January 2011

Accepted March 2011

Final acceptance in revised form April 2011 